\title{
SINGULAR RELAXATION MODULI AND SMOOTHING IN THREE-DIMENSIONAL VISCOELASTICITY
}

\author{
WOLFGANG DESCH AND RONALD GRIMMER
}

\begin{abstract}
We develop a semigroup setting for linear viscoelasticity in threedimensional space with tensor-valued relaxation modulus and give a criterion on the relaxation kernel for differentiability and analyticity of the solutions. The method is also extended to a simple problem in thermoviscoelasticity.
\end{abstract}

\section{INTRODUCTION}

We consider smoothing properties of systems of linear integral and differential equations in Hilbert spaces. Our equations occur naturally as an abstract setting for the equations of motion of a linearly viscoelastic body in threedimensional space.

There is extensive literature concerning the effects of viscoelasticity on propagation and nonpropagation of discontinuities. Smooth convolution kernels with one derivative bounded at 0 yield hyperbolic behavior in the sense that singularities are propagated much as in the purely elastic case, although they are exponentially damped (e.g., see $[1,6,7,9,10,12,17,23,25])$. However, recent literature indicates that many materials may be better described by equations involving singular kernels, such as fractional derivative models [2, 3, 26]. In [31] it is observed that such kernels can yield differentiable and even $C^{\infty}$ solutions. It is known that strong singularities at 0 will even yield analytic solution operators, e.g., [18]. In [21] it is shown that smoothing occurs if the singularity of the kernel at 0 is not weaker than logarithmic. If the singularity is stronger than logarithmic, then the solution is $C^{\infty}$. In [29] a method is developed to treat abstract integrodifferential equations with singular kernels in Banach space. In [30] a frequency domain criterion on the kernel for smoothing is given. The asymptotic ratio of the real and imaginary parts of the Laplace transform of the kernel turns out to be the crucial quantity governing smoothing. A related criterion for certain matrix-valued kernels is discussed in [11]. Other aspects of singular kernels have been studied, such as compactness of the solution operator [19], essential stability of the system [13], and effects of boundary feedback

Received by the editors February 8, 1988.

1980 Mathematics Subject Classification (1985 Revision). Primary 45K05, 73F99; Secondary 73D15, 34G10.

Supported within the U. S.-Austrian Cooperative Research Program, by National Science Foundation DMS-8701552, and by Fonds zur Förderung der Wissenschaftlichen Forschung P5691. 
stabilization and destabilization [20]. A thorough discussion of singular kernels and hyperbolicity is found in [32].

Most of the work mentioned above is confined to scalar kernels. To handle viscoelasticity in three-dimensional space, one has to consider tensor-valued relaxation moduli. In this paper we develop an abstract setting pertinent to this case and give a smoothing criterion similar to that of [30]. The formulation of our equations is somewhat unusual; they are a literal translation of the equation of momentum and the constitutive equation into abstract equations in Hilbert space. Basically, our method is very closely related to that in [30]. Both rely on finding logarithmic estimates for the Laplace transform of the solution for large imaginary parts. The transition from scalars to operators, replacing real and imaginary parts by self-adjoint and skew-adjoint parts, makes the results as well as the proofs look more technical. On the other hand, we introduce a semigroup setting similar to that in [13] to make the problem accessible to an abstract criterion for differentiability of semigroups ([27, 28], where one finds a detailed discussion of differentiable semigroups). This theorem requires only estimates on the imaginary axis rather than on a curve in the left half-plane; thus some of the work is considerably simplified.

Semigroup settings for integrodifferential equations are quite common $[4,8$, $24,33]$. Some of these settings require state spaces constructed particularly to the special kernels $[8,13,33]$. It seems that this is necessary in any case where singular kernels are to be treated. We choose a forcing function setting rather than a history space, because obtaining a differentiable semigroup while one carries on some nonsmooth history of the velocity field seems unlikely. It is somewhat unsatisfactory that our state space and its norm have no intuitive physical meaning (such as energy). Also, this construction is restricted to completely monotonic kernels, while physical considerations would allow for more general relaxation moduli. However, we feel that the latter restriction is not a serious one; in particular, fractional derivative kernels are completely monotone.

The paper is organized as follows: $\S 2$ sets up the abstract equations and provides the semigroup setting. $\S 3$ proves the smoothing criterion, and $\S 4$ gives the application to the equations of viscoelasticity. Finally, in $\S 5$ we sketch how our methods can be modified to treat some problems in linear thermoviscoelasticity.

\section{SEMIGROUP SETTING}

We consider the abstract problem

$$
\begin{gathered}
R v^{\prime}(t)=-D \sigma(t) \\
\sigma(t)=\int_{-\infty}^{t} A(t-s) D^{*} v(s) d s
\end{gathered}
$$


Here the prime denotes the derivative with respect to $t, v$ is supposed to be a continuous function on $[0, \infty)$ with values in some Hilbert space $Y$, and $\sigma$ takes values in a Hilbert space $X$.

Our assumptions are:

Hypothesis D. $D: X \supseteq \operatorname{dom} D \rightarrow Y$ is a closed, densely defined linear operator, and $D^{*}: Y \supseteq \operatorname{dom} D^{*} \rightarrow X$ is its adjoint.

Hypothesis R. $R: Y \rightarrow Y$ is a bounded, continuously invertible, positive definite linear operator.

Hypothesis A. For each $t>0, A(t)$ is a bounded linear operator on $X . A$ is completely monotonic in the following sense: There exist an operator-valued function $G$ and a nondecreasing function $\nu:[0, \infty) \rightarrow[0, \infty)$ such that for each $x \in X$

$$
A(t) x=\int_{0}^{\infty} e^{-\zeta t} G(\zeta) x d \nu(\zeta)
$$

where for each $\zeta>0 G(\zeta)$ is a bounded, self-adjoint, positive semidefinite linear operator on $X$, and for each $x \in X$ and $t>0$ the function $e^{-\zeta t} G(\zeta) x$ is (Bochner-) integrable with respect to $\nu$. Moreover, we assume that $\|A\|$ is integrable on $[0,1]$ and for some $t_{0}>0 A\left(t_{0}\right)$ is continuously invertible.

Let $L_{\nu}^{2}((0, \infty), X)$ be the space of $X$-valued $L^{2}$-functions with respect to the measure $d \nu$, endowed with the scalar product

$$
\langle\phi, \psi\rangle=\int_{0}^{\infty}\langle\phi(\zeta), \psi(\zeta)\rangle d \nu(\zeta) .
$$

(This space is very closely related to an $L^{2}$-space $L_{G}^{2}$ with respect to the operator-valued measure $G(\zeta) d \nu(\zeta)$; namely if $\phi \in L_{\nu}^{2}$, then $G^{-1 / 2} \phi \in L_{G}^{2}$. Actually the whole problem can also be set in terms of this space. For a discussion of this type of space see [35] and the references therein.)

By Hypothesis A we can-at least formally-rewrite the equation for $\sigma$ :

$$
\begin{aligned}
\sigma(t) & =\int_{-\infty}^{t} A(t-s) D^{*} v(s) d s \\
& =\int_{-\infty}^{t} \int_{0}^{\infty} e^{-\zeta(t-s)} G(\zeta) D^{*} v(s) d \nu(\zeta) d s=\int_{0}^{\infty} G^{1 / 2}(\zeta) \phi(t, \zeta) d \nu(\zeta),
\end{aligned}
$$

where $\phi(t, \zeta)=G^{1 / 2}(\zeta) \int_{-\infty}^{t} e^{-\zeta(t-s)} D^{*} v(s) d s$. This yields a simple differential equation for $\phi$ :

$$
\frac{\partial \phi}{\partial t}(t, \zeta)=-\zeta \phi(t, \zeta)+G^{1 / 2}(\zeta) D^{*} v(t)
$$

If the history of $v$ is such that $\phi(0,.) \in L_{\nu}^{2}$, we may rewrite (2.1) formally as

$$
\begin{aligned}
R v^{\prime}(t) & =-D \int_{0}^{\infty} G^{1 / 2}(\zeta) \phi(t, \zeta) d \nu(\zeta), \\
\phi^{\prime}(t, \zeta) & =-\zeta \phi(t, \zeta)+G^{1 / 2}(\zeta) D^{*} v(t), \quad(t \geq 0)
\end{aligned}
$$

with given initial data $v(0)$ and $\phi(0, \zeta)$. 
(2.2) is then treated as an abstract Cauchy problem in $\mathscr{X}=Y \times L_{\nu}^{2}$, the state of the system being the pair $(v(t), \phi(t,)$.$) . As a scalar product in \mathscr{X}$ we use $\langle(v, \phi),(w, \psi)\rangle=\langle v, R w\rangle+\langle\phi, \psi\rangle$. We define an operator $\mathscr{A}$ on

$$
\begin{aligned}
\operatorname{dom} \mathscr{A}=\left\{(v, \phi) \in \mathscr{X}: v \in \operatorname{dom} D^{*}, \zeta \phi(\zeta)-G^{1 / 2}(\zeta) D^{*} v \in L_{\nu}^{2},\right. \\
\left.\qquad \int_{0}^{\infty} G^{1 / 2}(\zeta) \phi(\zeta) d \nu(\zeta) \in \operatorname{dom} D\right\}
\end{aligned}
$$

by

$$
\mathscr{A}(v, \phi)=\left(-R^{-1} D \int_{0}^{\infty} G^{1 / 2}(\zeta) \phi(\zeta) d \nu(\zeta),-\zeta \phi(\zeta)+G^{1 / 2}(\zeta) D^{*} v\right)
$$

The integral $\int_{0}^{\infty} G^{1 / 2}(\zeta) \phi(\zeta) d \nu(\zeta)$ is taken in the weak sense. For a justification see Lemma 2.4 below.

We show in this section:

Theorem 2.1. $\mathscr{A}$ generates a $C_{0}$-semigroup $\mathscr{S}(t)$ of contractions on $\mathscr{X}$.

This implies wellposedness of (2.2) in the following sense (see [13]):

\section{Corollary 2.2.}

(a) Suppose that $v:(-\infty, 0] \rightarrow \operatorname{dom} D^{*}$ is given such that

$$
\phi_{0}(\zeta)=G^{1 / 2}(\zeta) \int_{-\infty}^{0} e^{\zeta t} D^{*} v(t) d t
$$

satisfies $\phi_{0} \in L_{\nu}^{2}, \zeta \phi_{0}-G^{1 / 2}(\zeta) D^{*} v(0) \in L_{\nu}^{2}$, and

$$
\int_{0}^{\infty} G^{1 / 2}(\zeta) \phi_{0}(\zeta) d \nu(\zeta)\left(=\int_{-\infty}^{0} A(-t) D^{*} v(t) d t\right) \in \operatorname{dom} D
$$

Then there exist unique solutions $v \in C^{1}([0, \infty), Y)$ and $\sigma \in C([0, \infty), X)$ such that (2.1) is satisfied for each $t>0$.

(b) $v(t) \in Y$ depends continuously on $v(0) \in Y$ and $\phi_{0} \in L_{\nu}^{2}$, uniformly for $t$ in compact intervals. Hence for all initial data $v(0) \in Y$ and $\phi_{0} \in$ $L_{\nu}^{2}$ a generalized solution $v \in C([0, \infty), Y)$ can be defined by approximation. (Notice, however, that $\sigma$ does not depend continuously on $v(0) \in Y$ and $\phi_{0} \in L_{\nu}^{2}$ unless $\int_{0}^{\infty} G(\zeta) d \nu(\zeta)$ is a bounded operator.)

(c) The Laplace transform of the generalized solution satisfies

$$
\lambda R \hat{v}(\lambda)-R v(0)=-D \hat{\sigma}(\lambda),
$$

where $\hat{\sigma}(\lambda)$ is defined by

$$
\hat{\sigma}(\lambda)=\widehat{A}(\lambda) D^{*} \hat{v}(\lambda)+\int_{0}^{\infty} \frac{1}{\lambda+\zeta} G^{1 / 2}(\zeta) \phi_{0}(\zeta) d \nu(\zeta)
$$


Here $\hat{A}$ denotes the Laplace transform of $A$; see Lemma 2.3 below. (In case (a), this $\hat{\sigma}$ is the Laplace transform of the solution $\sigma$.)

Proof. (a) Put $(v(t), \phi(t,))=.\mathscr{S}(t)\left(v(0), \phi_{0}\right)$. As the initial data have been constructed so that $\left(v(0), \phi_{0}\right) \in \operatorname{dom} \mathscr{A},(v(t), \phi(t))$ lies in dom $\mathscr{A}$, depending continuously on $t . \sigma(t)=\int_{0}^{\infty} G^{1 / 2}(\zeta) \phi(t, \zeta) d \nu(\zeta)$ is well defined (see Lemma 2.4 below). Equation (2.1) follows from the fact that $\left(v^{\prime}(t), \phi^{\prime}(t)\right)=$ $\mathscr{A}(v(t), \phi(t))$.

(b) is evident since the operators $\mathscr{S}(t)$ are uniformly bounded for $t$ in compact intervals.

(c) By standard semigroup theory the generalized solution $(v(t), \phi(t))=$ $\mathscr{S}(t)\left(v(0), \phi_{0}\right)$ satisfies $(\lambda-\mathscr{A})(\hat{v}(\lambda), \hat{\phi}(\lambda))=\left(v(0), \phi_{0}\right)$; i.e., $\lambda \hat{v}(\lambda)+$ $R^{-1} D \int_{0}^{\infty} G^{1 / 2}(\zeta) \hat{\phi}(\lambda, \zeta) d \nu(\zeta)=v(0)$, and $(\lambda+\zeta) \hat{\phi}(\lambda, \zeta)-G^{1 / 2}(\zeta) D^{*} \hat{v}(\lambda)=$ $\phi_{0}(\zeta)$. The latter equation yields

$$
\begin{aligned}
\int_{0}^{\infty} G^{1 / 2}(\zeta) \hat{\phi} & (\lambda, \zeta) d \nu(\zeta) \\
& =\int_{0}^{\infty} \frac{1}{\lambda+\zeta} G^{1 / 2}(\zeta) \phi_{0}(\zeta) d \nu(\zeta)+\widehat{A}(\lambda) D^{*} \hat{v}(\lambda)=\hat{\sigma}(\lambda)
\end{aligned}
$$

The rest of this section is devoted to the proof of Theorem 2.1, which is given by several lemmas. We start out with some technical details about the Laplace transform of $A$ :

\section{Lemma 2.3.}

(a) $\|A(t)\|$ is nonincreasing. In particular, for any $\lambda \in \mathbf{C}$ with $\operatorname{Re} \lambda>0, a$ continuous linear operator $\widehat{A}(\lambda)$ is defined in $X$ by the Bochner integral

$$
\widehat{A}(\lambda) x=\int_{0}^{\infty} e^{-\lambda t} A(t) x d t
$$

(b) For all $x \in X$ and $\lambda>0$, the function $\zeta \rightarrow(\lambda+\zeta)^{-1 / 2} G^{1 / 2}(\zeta) x$ is in $L_{\nu}^{2}((0, \infty), X)$, and

$$
\int_{0}^{\infty} \frac{1}{\lambda+\zeta}\left\|G^{1 / 2}(\zeta) x\right\|^{2} d \nu(\zeta) \leq\|\widehat{A}(\lambda)\|\|x\|^{2} .
$$

(c) For all $\lambda \in \mathbf{C} \backslash(-\infty, 0]$, a continuous linear operator is defined on $X$ by the weak integral

$$
\widehat{A}(\lambda) x=\int_{0}^{\infty} \frac{1}{\lambda+\zeta} G(\zeta) x d \nu(\zeta),
$$

and it is the analytic extension of $\hat{A}$ as it is defined in (a).

(d) For all $\lambda>0$ a continuous linear operator is defined on $X$ by the weak integral

$$
\int_{0}^{\infty} \frac{1}{(\lambda+\zeta)^{2}} G(\zeta) x d \nu(\zeta)
$$


(e) For all $\lambda>0, \hat{A}(\lambda)$ and $\int_{0}^{\infty}\left(1 /(\lambda+\zeta)^{2}\right) G(\zeta) d \nu(\zeta)$ are continuously invertible.

Proof. (a) Since for $s<t$ and all $x \in X$

$$
\begin{aligned}
\langle x, A(t) x\rangle & =\int_{0}^{\infty} e^{-\zeta t}\langle x, G(\zeta) x\rangle d \nu(\zeta) \leq \int_{0}^{\infty} e^{-\zeta s}\langle x, G(\zeta) x\rangle d \nu(\zeta) \\
& =\langle x, A(s) x\rangle
\end{aligned}
$$

we have that $A(t)$ and $A(s)-A(t)$ are positive semidefinite operators, whence $\|A(t)\| \leq\|A(s)\|$.

(b)

$$
\begin{aligned}
\int_{0}^{\infty} & \frac{1}{\lambda+\zeta}\left\langle G^{1 / 2}(\zeta) x, G^{1 / 2}(\zeta) x\right\rangle d \nu(\zeta) \\
& =\int_{0}^{\infty} \int_{0}^{\infty} e^{-\lambda t} e^{-\zeta t}\langle x, G(\zeta) x\rangle d t d \nu(\zeta) \\
& =\int_{0}^{\infty} \int_{0}^{\infty} e^{-\lambda t} e^{-\zeta t}\langle x, G(\zeta) x\rangle d \nu(\zeta) d t=\int_{0}^{\infty} e^{-\lambda t}\langle x, A(t) x\rangle d t \\
& =\langle x, \widehat{A}(\lambda) x\rangle .
\end{aligned}
$$

The interchange of order of integration is correct since

$$
\int_{0}^{\infty} e^{-\lambda t} \int_{0}^{\infty} e^{-\zeta t}\langle x, G(\zeta) x\rangle d \nu(\zeta) d t
$$

converges absolutely.

(c) Fix $x \in X$. For any $y \in X$

$$
\begin{aligned}
& \mid \int_{0}^{\infty} \frac{1}{\lambda+\zeta}\langle y, G(\zeta) x\rangle d \nu(\zeta)\left|\leq \int_{0}^{\infty} \frac{1}{|\lambda+\zeta|}\right|\left\langle G^{1 / 2}(\zeta) y, G^{1 / 2}(\zeta) x\right\rangle \mid d \nu(\zeta) \\
& \quad \leq\left[\int_{0}^{\infty} \frac{M}{1+\zeta}\left\|G^{1 / 2}(\zeta) y\right\|^{2} d \nu(\zeta)\right]^{1 / 2}\left[\int_{0}^{\infty} \frac{M}{1+\zeta}\left\|G^{1 / 2}(\zeta) x\right\|^{2} d \nu(\zeta)\right]^{1 / 2} \\
& \quad \leq M\|\widehat{A}(\lambda)\|\|y\|\|x\| \quad \text { by (b). }
\end{aligned}
$$

Thus $\int_{0}^{\infty}(1 /(\lambda+\zeta))\langle y, G(\zeta) x\rangle d \nu(\zeta)$ exists and depends continuously on $y$ and $x$. Obviously, for fixed $x$ and $y$ it depends analytically on $\lambda$. Utilizing repeatedly the fact that a family of bounded linear operators depending weakly analytically on a parameter $\lambda$ depends in fact analytically in the sense of operator topology, we infer analyticity of $\widehat{A}$. For $\lambda>0$, the definitions of $\widehat{A}(\lambda)$ in (a) and (c) are equivalent, since

$$
\begin{aligned}
\int_{0}^{\infty} \frac{1}{\lambda+\zeta}\langle y, G(\zeta) x\rangle d \nu(\zeta)=\int_{0}^{\infty} \int_{0}^{\infty} e^{-(\lambda+\zeta) t}\langle y, G(\zeta) x\rangle d \nu(\zeta) \\
=\int_{0}^{\infty} \int_{0}^{\infty} e^{-(\lambda+\zeta) t}\langle y, G(\zeta) x\rangle d \nu(\zeta)=\int_{0}^{\infty} e^{-\lambda t}\langle y, A(t) x\rangle d t
\end{aligned}
$$


(d)

$$
\begin{aligned}
& \int_{0}^{\infty} \frac{1}{(\lambda+\zeta)^{2}}|\langle y, G(\zeta) x\rangle| d \nu(\zeta) \leq \int_{0}^{\infty} \frac{M}{\lambda+\zeta}|\langle y, G(\zeta) x\rangle| d \nu(\zeta) \\
& \quad \leq M\left[\int_{0}^{\infty} \frac{1}{\lambda+\zeta}\left\|G^{1 / 2}(\zeta) y\right\|^{2} d \nu(\zeta)\right]^{1 / 2}\left[\int_{0}^{\infty} \frac{1}{\lambda+\zeta}\left\|G^{1 / 2}(\zeta) x\right\|^{2} d \nu(\zeta)\right]^{1 / 2} \\
& \leq M\|\hat{A}(\lambda)\|\|y\|\|x\| \quad \text { with some constant } M .
\end{aligned}
$$

(e) All we have to show is that for some constant $M$ and all $x \in X\langle x, \widehat{A}(\lambda) x\rangle$ $\geq M\left\langle x, A\left(t_{0}\right) x\right\rangle$. Then positivity and invertibility of $A\left(t_{0}\right)$ imply invertibility of $\widehat{A}(\lambda)$.

$$
\begin{aligned}
\langle x, \hat{A}(\lambda) x\rangle & =\int_{0}^{\infty} \frac{1}{\lambda+\zeta}\langle x, G(\zeta) x\rangle d \nu(\zeta) \geq M \int_{0}^{\infty} e^{-t_{0} \zeta}\langle x, G(\zeta) x\rangle d \nu(\zeta) \\
& =M\left\langle x, A\left(t_{0}\right) x\right\rangle .
\end{aligned}
$$

The same argument works for the operator defined in (d).

Lemma 2.4. If for some $w \in X$ and $\phi \in L_{\nu}^{2}((0, \infty), X)$ the function

$$
\psi(\zeta)=\zeta \phi(\zeta)-G^{1 / 2}(\zeta) w
$$

lies in $L_{\nu}^{2}((0, \infty), X)$, then the weak integral

$$
\int_{0}^{\infty} G^{1 / 2}(\zeta) \phi(\zeta) d \nu(\zeta)
$$

exists and depends continuously on $w \in X$ and $\phi$ and $\psi \in L_{\nu}^{2}((0, \infty), X)$.

Proof. Fix some $\lambda>0$. For any $y \in X$,

$$
\begin{aligned}
\int_{0}^{\infty} & \left|\left\langle y, G^{1 / 2}(\zeta) \phi(\zeta)\right\rangle\right| d \nu(\zeta)=\int_{0}^{\infty} \frac{1}{\lambda+\zeta}\left|\left\langle G^{1 / 2}(\zeta) y,(\lambda+\zeta) \phi(\zeta)\right\rangle\right| d \nu(\zeta) \\
= & \int_{0}^{\infty} \frac{1}{\lambda+\zeta}\left|\left\langle G^{1 / 2}(\zeta) y, \lambda \phi(\zeta)+\psi(\zeta)+G^{1 / 2}(\zeta) w\right\rangle\right| d \nu(\zeta) \\
\leq & \lambda^{1 / 2} \int_{0}^{\infty}(\lambda+\zeta)^{-1 / 2}\left|\left\langle G^{1 / 2}(\zeta) y, \phi(\zeta)\right\rangle\right| d \nu(\zeta) \\
& +\lambda^{-1 / 2} \int_{0}^{\infty}(\lambda+\zeta)^{-1 / 2}\left|\left\langle G^{1 / 2}(\zeta) y, \psi(\zeta)\right\rangle\right| d \nu(\zeta) \\
& +\int_{0}^{\infty}(\lambda+\zeta)^{-1}\left|\left\langle G^{1 / 2}(\zeta) y, G^{1 / 2}(\zeta) w\right\rangle\right| d \nu(\zeta) \\
\leq & \lambda^{1 / 2}\|\widehat{A}(\lambda)\|^{1 / 2}\|y\|\|\phi\|+\lambda^{-1 / 2}\|\widehat{A}(\lambda)\|^{1 / 2}\|y\|\|\psi\|+\|\widehat{A}(\lambda)\|\|y\|\|w\| .
\end{aligned}
$$

Lemma 2.5. $\mathscr{A}$ is densely defined in $\mathscr{Z}$.

Proof. Given $(y, \psi) \in Y \times L_{\nu}^{2}$ and $\varepsilon>0$ we have to find $(v, \phi) \in \operatorname{dom} \mathscr{A}$ such that $\|(v, \phi)-(y, \psi)\| \leq \varepsilon$. As $D^{*}$ is densely defined, we can choose $v \in \operatorname{dom} D^{*}$ such that $\|v-y\| \leq \varepsilon / 4$. Next we choose $T$ sufficiently large 
such that $\int_{T}^{\infty}\langle\psi(\zeta), \psi(\zeta)\rangle d \nu(\zeta)<\varepsilon^{2} / 16$ and $\int_{T}^{\infty} \zeta^{-2}\left\langle D^{*} v, G(\zeta) D^{*} v\right\rangle d \nu(\zeta)<$ $\varepsilon^{2} / 16$; define

$$
\pi(\zeta)= \begin{cases}\psi(\zeta) & \text { for } \zeta \leq T, \\ \zeta^{-1} G^{1 / 2}(\zeta) D^{*} v & \text { for } \zeta>T .\end{cases}
$$

Then $\|\pi-\psi\| \leq \varepsilon / 2$. Now we fix some $\lambda>0$ and put $\phi(\zeta)=\pi(\zeta)+$ $(\lambda+\zeta)^{-2} G^{1 / 2}(\zeta) w$ with some small $w$ such that $\int_{0}^{\infty}(\lambda+\zeta)^{-4}\langle w, G(\zeta) w\rangle d \nu(\zeta)$ $\leq \varepsilon^{2} / 16$. To obtain that $(v, \phi) \in \operatorname{dom} \mathscr{A}$ we need $G^{1 / 2}(\zeta) D^{*} v-\zeta \phi \in L_{\nu}^{2}$, which holds as $\zeta(\lambda+\zeta)^{-2} G^{1 / 2}(\zeta) w \in L_{\nu}^{2}$ for any $w$ and $\zeta \pi-G^{1 / 2}(\zeta) D^{*} v$ has compact support. Furthermore, we require that

$$
\begin{aligned}
& \int_{0}^{\infty} G^{1 / 2}(\zeta) \phi(\zeta) d \nu(\zeta) \\
&=\int_{0}^{\infty} G^{1 / 2}(\zeta) \pi(\zeta) d \nu(\zeta)+\int_{0}^{\infty}(\lambda+\zeta)^{-2} G(\zeta) w d \nu(\zeta) \in \operatorname{dom} D
\end{aligned}
$$

As $\operatorname{dom} D$ is dense in $X$ we can find arbitrarily small $h \in X$ such that $\int_{0}^{\infty} G^{1 / 2}(\zeta) \pi(\zeta) d \nu(\zeta)+h \in \operatorname{dom} D$. Since $\int_{0}^{\infty}(\lambda+\zeta)^{-2} G(\zeta) d \nu(\zeta)$ is continuously invertible, we can put $w=\left(\int_{0}^{\infty}(\lambda+\zeta)^{-2} G(\zeta) d \nu(\zeta)\right)^{-1} h$ with some $h$ which is small enough so that the required estimate for $w$ is satisfied.

Lemma 2.6. $\mathscr{A}$ is dissipative.

Proof. Take $(v, \phi) \in \operatorname{dom} \mathscr{A}$. Then

$$
\begin{aligned}
\operatorname{Re}\langle(v, \phi), \mathscr{A}(v, \phi)\rangle= & \operatorname{Re}\left\langle v,-R R^{-1} D \int_{0}^{\infty} G^{1 / 2}(\zeta) \phi(\zeta) d \nu(\zeta)\right\rangle \\
& +\operatorname{Re} \int_{0}^{\infty}\left\langle\phi(\zeta), G^{1 / 2}(\zeta) D^{*} v-\zeta \phi(\zeta)\right\rangle d \nu(\zeta) \\
= & -\operatorname{Re}\left\langle D^{*} v, \int_{0}^{\infty} G^{1 / 2}(\zeta) \phi(\zeta) d \nu(\zeta)\right\rangle \\
& +\operatorname{Re}\left\langle\int_{0}^{\infty} G^{1 / 2}(\zeta) \phi(\zeta) d \nu(\zeta), D^{*} v\right\rangle \\
& -\int_{0}^{\infty} \zeta\langle\phi(\zeta), \phi(\zeta)\rangle d \nu(\zeta) \\
= & -\int_{0}^{\infty} \zeta\langle\phi(\zeta), \phi(\zeta)\rangle d \nu(\zeta) \leq 0 .
\end{aligned}
$$

Lemma 2.7. For $\operatorname{Re} \lambda>0,(\lambda-\mathscr{A})^{-1}$ exists as a bounded linear operator on $\mathscr{Z}$.

Proof. Since $\mathscr{A}$ is dissipative it is sufficient to show that for some $\lambda>0$ the range of $\lambda-\mathscr{A}$ is the whole space $\mathscr{X}$. Thus for given $(y, \psi) \in \mathscr{X}$ we have to find a solution $(v, \phi)$ to $(\lambda-\mathscr{A})(v, \phi)=(y, \psi)$, i.e.,

$$
\begin{gathered}
\lambda v+R^{-1} D \int_{0}^{\infty} G^{1 / 2}(\zeta) \phi(\zeta) d \nu(\zeta)=y, \\
\lambda \phi(\zeta)-G^{1 / 2}(\zeta) D^{*} v+\zeta \phi(\zeta)=\psi(\zeta) .
\end{gathered}
$$


The latter equation yields immediately

$$
\phi(\zeta)=(\lambda+\zeta)^{-1} \psi(\zeta)+(\lambda+\zeta)^{-1} G^{1 / 2}(\zeta) D^{*} v
$$

Given $v \in \operatorname{dom} D^{*}$, such $\phi$ lies evidently in $L_{\nu}^{2}$, and since

$$
\left[\zeta(\lambda+\zeta)^{-1}-1\right] G^{1 / 2}(\zeta) D^{*} v=-\lambda(\lambda+\zeta)^{-1} G^{1 / 2}(\zeta) D^{*} v
$$

also $\zeta \phi-G^{1 / 2}(\zeta) D^{*} v \in L_{\nu}^{2}$. So we have only to determine a suitable $v$. Putting

$$
\begin{aligned}
& \tilde{\psi}(\lambda)=\int_{0}^{\infty}(\lambda+\zeta)^{-1} G^{1 / 2}(\zeta) \psi(\zeta) d \nu(\zeta), \\
& z=\int_{0}^{\infty} G^{1 / 2}(\zeta) \phi(\zeta) d \nu(\zeta)=\widehat{A}(\lambda) D^{*} v+\tilde{\psi}(\lambda),
\end{aligned}
$$

we have to find $v \in \operatorname{dom} D^{*}$ and $z \in \operatorname{dom} D$ such that

$$
\begin{gathered}
\lambda z-\lambda \widehat{A}(\lambda) D^{*} v=\lambda \tilde{\psi}(\lambda), \\
\lambda v+R^{-1} D z=y .
\end{gathered}
$$

We put $w=\lambda \tilde{\psi}(\lambda)$. We consider the system (2.7), (2.8) in the Hilbert space $X \times Y$, with the scalar product.

$$
\langle(z, v),(\tilde{z}, \tilde{v})\rangle=\left\langle z, \lambda^{-1} \hat{A}(\lambda)^{-1} \tilde{z}\right\rangle+\langle v, R \tilde{v}\rangle .
$$

For any solution we have

$$
\lambda\langle(z, v),(z, v)\rangle-\operatorname{Re}\langle(z, v)(w, y)\rangle=\operatorname{Re}\left\langle(z, v),\left(\lambda \widehat{A}(\lambda) D^{*} v,-R^{-1} D z\right)\right\rangle=0,
$$

hence $\|(z, v)\| \leq \lambda^{-1}\|(w, y)\|$. This implies that $(z, v)$ depends continuously on $(w, y)$, and by closedness of $D$ and $D^{*}$ we infer that the set of all $(w, y)$ that admit solutions to (2.7), (2.8) is a closed subspace of $X \times Y$. Now let $(w, y)$ be orthogonal to this set; i.e., for all $z \in \operatorname{dom} D$ and $v \in \operatorname{dom} D^{*}$, $\left(\lambda z-\lambda \widehat{A}(\lambda) D^{*} v, \lambda v+R^{-1} D z\right) \perp(w, y)$. This says

$$
\left\langle z, \widehat{A}(\lambda)^{-1} w\right\rangle-\left\langle D^{*} v, w\right\rangle+\langle\lambda v, R y\rangle+\langle D z, y\rangle=0 .
$$

If we put $v=0$, we obtain for arbitrary $z \in \operatorname{dom} D,\left\langle z, \widehat{A}(\lambda)^{-1} w\right\rangle+\langle D z, y\rangle=$ 0 ; thus $y \in \operatorname{dom} D^{*}, D^{*} y=-\widehat{A}(\lambda)^{-1} w$. Similarly, $w \in \operatorname{dom} D, D w=\lambda R y$. Now we use $z=w$ and $v=y$ in (2.9) to obtain

$$
\left\langle w, \widehat{A}(\lambda)^{-1} w\right\rangle+\left\langle\widehat{A}(\lambda)^{-1} w, w\right\rangle+\langle\lambda y, R y\rangle+\langle\lambda R y, y\rangle=0 .
$$

As $\widehat{A}(\lambda)^{-1}$ and $R$ are positive definite, this implies that $(w, y)=0$. Consequently, (2.7) and (2.8) admit solutions for any $y \in Y, w=\lambda \tilde{\psi}(\lambda) \in X$. This proves the lemma.

Theorem 2.1 follows immediately from Lemmas $2.5-2.7$, since a densely defined $m$-dissipative linear operator generates a $C_{0}$-semigroup of contractions. 


\section{Smoothing}

We call a $C_{0}$-semigroup $\mathscr{S}(t)$ generated by $\mathscr{A}$ in $\mathscr{X}$ differentiable if for each $t>0 \mathscr{S}(t)$ maps $\mathscr{X}$ into $\operatorname{dom} \mathscr{A}$. If this is true only for $t>t_{0}$ with some $t_{0}>0$, we call the semigroup eventually differentiable. Thus differentiability implies that the trajectories $t \rightarrow \mathscr{S}(t) x$ are $C^{\infty}$ in $(0, \infty)$ for all $x \in \mathscr{Z}$, while for eventually differentiable semigroups the trajectories gain smoothness gradually. As in usual applications, dom $\mathscr{A}$ consists of functions with certain differentiability properties; differentiability of the semigroup also implies some spatial smoothing. Differentiable semigroups are discussed in detail in [27, 28]. There the reader can find the following criterion for differentiability:

Differentiability Criterion. Let $\mathscr{S}(t)$ be a $C_{0}$-semigroup generated by $\mathscr{A}$, and suppose that $\alpha \in \mathbf{R}$ is such that for sufficiently large $|\beta|(\alpha+i \beta-\mathscr{A})^{-1}$ exists.

(a) If $\lim \sup _{|\beta| \rightarrow \infty} \log |\beta|\left\|(\alpha+i \beta-\mathscr{A})^{-1}\right\|<\infty$, then $\mathscr{S}(t)$ is eventually differentiable.

(b) If $\lim \sup _{|\beta| \rightarrow \infty} \log |\beta|\left\|(\alpha+i \beta-\mathscr{A})^{-1}\right\|=0$, then $\mathscr{S}(t)$ is differentiable.

(c) If $\lim \sup _{|\beta| \rightarrow \infty}|\beta|\left\|(\alpha+i \beta-\mathscr{A})^{-1}\right\|<\infty$, then $\mathscr{S}(t)$ is analytic.

Proof. (a) and (b) are found in [27]. (c) can easily be verified using the standard characterization of generators of analytic semigroups (see, e.g., [28]) and estimating the Neumann series of $(\lambda-\mathscr{A})^{-1}$ for $\lambda$ in a neighborhood of $\alpha+i \beta$.

We return now to the semigroup $\mathscr{S}(t)$ constructed in the previous section. The main result of this section is that this semigroup satisfies

Theorem 3.1. With the notation and assumptions of $\S 2$, let $\lambda \widehat{A}(\lambda)=U(\lambda)+$ $i V(\lambda)$ with self-adjoint operators $U$ and $V$. Suppose that for some function $\gamma:(0, \infty) \rightarrow(0, \infty)$ and all $\beta>0$ the operator $\beta V(i \beta)-\gamma(\beta) U(i \beta)$ is positive semidefinite.

(a) If $\lim \sup _{\beta \rightarrow \infty} \log (\beta) / \gamma(\beta)=0$, then $\mathscr{S}(t)$ is differentiable.

(b) If lim $\sup _{\beta \rightarrow \infty} \log (\beta) / \gamma(\beta)<\infty$, then $\mathscr{S}(t)$ is eventually differentiable.

(c) If $\lim \sup _{\beta \rightarrow \infty} \beta / \gamma(\beta)<\infty$, then $\mathscr{S}(t)$ is analytic.

In terms of the integrodifferential system (2.1) this means

Corollary 3.2. Let $\gamma$ be defined as in the theorem above, and let $v$ be given on $(-\infty, 0]$ such that $\phi_{0}$ defined in Corollary 2.2 is in $L_{\nu}^{2}$.

(a) If $\lim \sup _{\beta \rightarrow \infty} \log (\beta) / \gamma(\beta)=0$ then there exist solutions $v, \sigma$ to (2.1) in the sense that $v \in C^{\infty}((0, \infty), Y), \sigma \in C^{\infty}((0, \infty), X)$, and (2.1) holds for all $t>0$.

(b) If $\lim \sup _{\beta \rightarrow \infty} \log (\beta) / \gamma(\beta)<\infty$, then the generalized solution $v$ to $(2.1)$ gains smoothness in $t$ gradually. For sufficiently large $t, \sigma(t)$ is defined such that (2.1) holds, and $\sigma(t)$ gains smoothness gradually. 
Proof. The only nontrivial parts of this corollary are the assertions concerning $\sigma$. As soon as $(v(t), \phi(t,).) \in \operatorname{dom} \mathscr{A}$, by Lemma 2.4

$$
\sigma(t)=\int_{0}^{\infty} G^{1 / 2}(\zeta) \phi(t, \zeta) d \nu(\zeta)
$$

is well defined and depends continuously on the graph norm of $(v(t), \phi(t,)$. with respect to $\mathscr{A}$.

The proof of the theorem is given by estimating $(i \beta-\mathscr{A})^{-1}$ for $\beta \rightarrow \infty$ and utilizing the differentiability criterion mentioned above. As we have only proved existence of $(\lambda-\mathscr{A})^{-1}$ for $\operatorname{Re} \lambda>0$, we will need the following simple observation:

Lemma 3.3. Let $\lambda$ be in the closure of the resolvent set of $\mathscr{A}$ and suppose that for some $M>0$ and each $x \in \operatorname{dom} \mathscr{A},\|x\| \leq M\|(\lambda-\mathscr{A}) x\|$. Then $(\lambda-\mathscr{A})^{-1}$ exists (and evidently $\left.\left\|(\lambda-\mathscr{A})^{-1}\right\| \leq M\right)$.

Proof. Let $\zeta$ be in the resolvent set of $\mathscr{A}$ such that $|\zeta-\lambda|<1 /(2 M)$. As $\|x\| \leq M\|(\lambda-\mathscr{A}) x\| \leq M\|(\zeta-\mathscr{A}) x\|+M|\lambda-\zeta|\|x\| \leq M\|(\zeta-\mathscr{A}) x\|+\|x\| / 2$, we infer that $\left\|(\zeta-\mathscr{A})^{-1}\right\| \leq 2 M$. This implies that the Neumann series $(\lambda-\mathscr{A})^{-1}=\sum_{j=0}^{\infty}(\zeta-\lambda)^{j}(\zeta-\mathscr{A})^{-j-1}$ converges, hence $(\lambda-\mathscr{A})^{-1}$ exists.

Lemma 3.4. With the notation of Theorem 3.1, for $\beta>0,(i \beta-\mathscr{A})^{-1}$ exists and $\left\|(i \beta-\mathscr{A})^{-1}\right\|=O(1 / \gamma(\beta)+1 / \beta)$ as $\beta \rightarrow \infty$.

Proof. In view of the lemma above, existence of $(i \beta-\mathscr{A})^{-1}$ is guaranteed once we can prove the appropriate estimate. Thus assume that $(v, \phi) \in \operatorname{dom} \mathscr{A}$ satisfies $(i \beta-\mathscr{A})(v, \phi)=(y, \psi)$ with $\|(y, \psi)\| \leq 1$, i.e.,

$$
\begin{gathered}
i \beta v+R^{-1} D \int_{0}^{\infty} G^{1 / 2}(\zeta) \phi(\zeta) d \nu(\zeta)=y, \\
i \beta \phi(\zeta)-G^{1 / 2}(\zeta) D^{*} v+\zeta \phi(\zeta)=\psi(\zeta) .
\end{gathered}
$$

Again we obtain

$$
\phi(\zeta)=(\zeta+i \beta)^{-1} \psi(\zeta)+(\zeta+i \beta)^{-1} G^{1 / 2}(\zeta) D^{*} v,
$$

hence

$$
i \beta v+R^{-1} D\left(\widehat{A}(i \beta) D^{*} v+\tilde{\psi}(i \beta)\right)=y,
$$

where

$$
\tilde{\psi}(i \beta)=\int_{0}^{\infty}(i \beta+\zeta)^{-1} G^{1 / 2}(\zeta) \psi(\zeta) d \nu(\zeta) .
$$

Multiplying (3.4) by $i \beta$ and taking inner products with $R v$ we obtain

$$
-\beta^{2}\langle v, R v\rangle+\left\langle D^{*} v, i \beta \widehat{A}(i \beta) D^{*} v\right\rangle+\left\langle D^{*} v, i \beta \tilde{\psi}(i \beta)\right\rangle-\langle v, i \beta R y\rangle=0 .
$$

Taking real and imaginary parts in (3.6) yields

$$
\left.-\beta^{2}\langle v, R v\rangle+\left\langle D^{*} v, U D^{*} v\right\rangle+\operatorname{Re}\left\langle D^{*} v, i \beta \tilde{\psi}\right\rangle-\operatorname{Re}\langle v, i \beta R y\rangle\right]=0,
$$




$$
\left\langle D^{*} v, V D^{*} v\right\rangle+\operatorname{Im}\left\langle D^{*} v, i \beta \tilde{\psi}\right\rangle-\operatorname{Im}\langle v, i \beta R y\rangle=0 .
$$

(We have suppressed the argument $(i \beta)$ in $U, V, \tilde{\psi}$.$) We need an estimate$ for $\tilde{\psi}$. We estimate for $x \in X$ :

$$
\begin{aligned}
|\langle x, \tilde{\psi}\rangle| & =\left|\left\langle x, \int_{0}^{\infty}(\zeta+i \beta)^{-1} G^{1 / 2}(\zeta) \psi(\zeta) d \nu(\zeta)\right\rangle\right| \\
& \leq\left[\int_{0}^{\infty}\left\langle x,|\zeta+i \beta|^{-2} G(\zeta) x\right\rangle d \nu(\zeta)\right]^{1 / 2}\left[\int_{0}^{\infty}\langle\psi(\zeta), \psi(\zeta)\rangle d \nu(\zeta)\right]^{1 / 2} \\
& =\left\langle x, \int_{0}^{\infty} \frac{1}{\beta^{2}+\zeta^{2}} G(\zeta) x d \nu(\zeta)\right\rangle^{1 / 2}\|\psi\| \\
& =\left\langle x, \frac{1}{i \beta} \int_{0}^{\infty} \frac{i \beta}{\beta^{2}+\zeta^{2}} G(\zeta) x d \nu(\zeta)\right\rangle^{1 / 2}\|\psi\| \\
& =\left[\beta^{-1} \operatorname{Im}\langle x, \widehat{A}(i \beta) x\rangle\right]^{1 / 2}\|\psi\|=\left[\beta^{-1} \operatorname{Im}\left\langle x, \frac{1}{i \beta}(U+i V) x\right\rangle\right]^{1 / 2}\|\psi\| \\
& =\left[\beta^{-2}\langle x, U x\rangle\right]^{1 / 2}\|\psi\| .
\end{aligned}
$$

Thus

$$
|\langle x, \tilde{\psi}(i \beta)\rangle| \leq \beta^{-1}\langle x, U x\rangle^{1 / 2}\|\psi\| .
$$

Now (3.7) yields

$$
\begin{aligned}
\beta^{2}\langle v & \left.+\frac{i}{2 \beta} y, R\left(v+\frac{i}{2 \beta} y\right)\right\rangle \\
& \leq\left\langle D^{*} v, U D^{*} v\right\rangle+\left\langle D^{*} v, U D^{*} v\right\rangle^{1 / 2}\|\psi\|+\beta^{2}\left\langle\frac{1}{2 \beta} y, \frac{1}{2 \beta} R y\right\rangle \\
& =O(1)\left[\left\langle D^{*} v, U D^{*} v\right\rangle+O(1)\right] .
\end{aligned}
$$

From (3.8) we obtain

$$
\begin{aligned}
\frac{\gamma(\beta)}{\beta} & \left\langle D^{*} v, U D^{*} v\right\rangle \leq\left\langle D^{*} v, V D^{*} v\right\rangle \leq \beta\left|\left\langle D^{*} v, \tilde{\psi}\right\rangle\right|+\operatorname{Im}\langle v, i \beta R y\rangle \\
& \leq O(1)\left\langle D^{*} v, U D^{*} v\right\rangle^{1 / 2}+\operatorname{Im}\left\langle v+\frac{i}{2 \beta} y, i \beta R y\right\rangle \quad(\text { by (3.9)) } \\
& \leq O(1)\left\langle D^{*} v, U D^{*} v\right\rangle^{1 / 2}+O(1)\left[\left\langle D^{*} v, U D^{*} v\right\rangle^{1 / 2}+O(1)\right] \quad(\text { by }(3.10)),
\end{aligned}
$$

hence

$$
\left\langle D^{*} v, U D^{*} v\right\rangle^{1 / 2}=O(\beta / \gamma(\beta)+1) .
$$

Again by (3.10) we have

$$
\|v\|=O(1 / \gamma(\beta)+1 / \beta) .
$$


To estimate $\phi(\zeta)=(\zeta+i \beta)^{-1} G^{1 / 2}(\zeta) D^{*} v+(\zeta+i \beta)^{-1} \psi(\zeta)$ notice that

$$
\begin{aligned}
\int_{0}^{\infty} & \left\langle\frac{1}{\zeta+i \beta} D^{*} v, \frac{1}{\zeta+i \beta} G(\zeta) D^{*} v\right\rangle d \nu(\zeta) \\
& =\int_{0}^{\infty}\left\langle D^{*} v, \frac{1}{\zeta^{2}+\beta^{2}} G(\zeta) D^{*} v\right\rangle d \nu(\zeta) \\
& =-\frac{1}{\beta} \operatorname{Im}\left\langle D^{*} v, \int_{0}^{\infty} \frac{\zeta-i \beta}{\zeta^{2}+\beta^{2}} G(\zeta) D^{*} v d \nu(\zeta)\right\rangle \\
& =\frac{1}{\beta^{2}} \operatorname{Re}\left\langle D^{*} v, i \beta \widehat{A}(i \beta) D^{*} v\right\rangle \\
& =\frac{1}{\beta^{2}}\left\langle D^{*} v, U D^{*} v\right\rangle=O\left(\frac{1}{\gamma^{2}}+\frac{1}{\beta^{2}}\right),
\end{aligned}
$$

and evidently $\|(1 /(i \beta+\zeta)) \psi\| \leq(1 / \beta)\|\psi\|=O(1 / \beta)$, so that

$$
\|\phi\|=O(1 / \gamma(\beta)+1 / \beta) \text {. }
$$

Lemma 3.4, inserted into the differentiability criterion, immediately yields Theorem 3.1.

\section{HomogeneOUS VisCoelastic MATERIALS}

In this section we show how small deformations of a viscoelastic body fit in the framework developed in the previous sections. We consider a homogeneous linearly viscoelastic body. (Homogeneity is not crucial but makes the notation somewhat less tedious.)

Let $\Omega \subset \mathbf{R}^{3}$ be a bounded domain with $C^{2}$-boundary, which describes a stress-free reference configuration of the body. $d x$ will denote the volume element, $x=\left(x_{1}, x_{2}, x_{3}\right)^{T}$ stands for particle coordinates. $\Gamma$ is the boundary of $\Omega$ with surface element $d S . n(x)$ is the outward normal unit vector at $x \in \Gamma$.

As usual, a prime denotes the derivative with respect to $t$, while spatial derivatives are abbreviated by $f_{, i}=\partial f / \partial x_{i}$. We use the following summation convention: Unless stated otherwise, every index appearing more than once in a term is a summation index.

Let $u(t, x)$ be the displacement of the particle $x$ at time $t, v=u^{\prime}$ the velocity field. $\varepsilon=\left(\varepsilon_{i j}\right)_{i, j=1 \ldots 3}$ is the strain, i.e., the symmetrized deformation gradient $\varepsilon_{i j}=\frac{1}{2}\left(u_{i, j}+u_{j, i}\right)$. We will use the rate of strain $\varepsilon_{i j}^{\prime}=\frac{1}{2}\left(v_{i, j}+v_{j, i}\right)$. $\sigma=\left(\sigma_{i j}\right)_{i, j=1 \cdots 3}$ is the stress. (Treating only small deformations, we need not distinguish between Cauchy stress and Piola-Kirchoff stress.)

We assume that the material satisfies the following constitutive equation:

$$
\sigma_{i j}(t, x)=\int_{-\infty}^{t} a_{i j k l}(t-s) \varepsilon_{k l}^{\prime}(s, x) d s
$$

(For the equations of linear viscoelasticity we refer to $[5,14,22]$. We remark that this constitutive equation, applied to a solid, describes the case that the 
material was initially at rest a long time back. The equation can easily be modified to describe a material that has been under constant strain for an infinitely long time. In this case, the equation needs to be written in terms of rate of strain and the strain itself, in a manner analogous to the scalar equation treated in [13]. The mathematical treatment of this equation and the results obtained are exactly the same as those for (4.1).)

For the relaxation kernel $a$ we assume:

Hypothesis a. $a_{i j k l}$ satisfies the following symmetry condition:

$$
a_{i j k l}=a_{j i k l}=a_{k l i j} \text {. }
$$

Moreover, there are a tensor-valued function $g=g_{i j k l}$ on $(0, \infty)$ and a nondecreasing function $\nu:[0, \infty) \rightarrow[0, \infty)$ such that

$$
a_{i j k l}(t)=\int_{0}^{\infty} e^{-\zeta t} g_{i j k l}(\zeta) d \nu(\zeta) .
$$

For all $\zeta, g(\zeta)$ is positive semidefinite in the sense that for all $\xi \in \mathbf{R}^{3 \times 3}$, $\xi_{i j} g_{i j k l}(\zeta) \xi_{k l} \geq 0$. a is assumed to be integrable on $(0,1)$, and for some $t_{0}>0, a\left(t_{0}\right)$ is positive definite. Of course, we have the

Momentum equation:

$$
\rho v_{i}^{\prime}(t, x)=\sigma_{i j, j}(t, x),
$$

where $\rho$ is the mass density in reference configuration.

Finally, we give energy-preserving

Boundary conditions: $\Gamma$ is the disjoint union of two parts $\Gamma_{0}, \Gamma_{1}$ such that the body is fixed at $\Gamma_{0}$ and stress-free at $\Gamma_{1}$, i.e.,

$$
\begin{gathered}
v(t, x)=0 \text { for } x \in \Gamma_{0}, \\
\sigma_{i j}(t, x) n_{j}(x)=0 \text { for } x \in \Gamma_{1} .
\end{gathered}
$$

( $\Gamma_{0}$ or $\Gamma_{1}$ may be empty.)

As state space for the velocity field $v(t)$ we take $Y=L^{2}\left(\Omega, \mathbf{R}^{3}\right)$ with the usual norm; for the stress field $\sigma(t)$ we put $X=L^{2}\left(\Omega, \mathbf{R}_{\text {sym }}^{3 \times 3}\right)$ with $\|\sigma\|^{2}=$ $\int_{\Omega} \sigma_{i j}(x) \sigma_{i j}(x) d x$. Here $\mathbf{R}_{\mathrm{sym}}^{3 \times 3}$ denotes the space of symmetric real $3 \times 3$ tensors.

To rewrite (4.2) as

$$
R v^{\prime}(t)=-D \sigma(t)
$$

we put $R=\rho$.id in $Y$, and let $D$ be defined on

$$
\operatorname{dom} D=\left\{\sigma \in X: \sigma_{i j, j} \in L^{2}(\Omega, \mathbf{R}) \text {, for } x \in \Gamma_{1}, \sigma_{i j}(x) n_{j}(x)=0\right\}
$$

by $(D \sigma)_{i}=-\sigma_{i j, j}$. (Derivatives are taken in the sense of distributions. The boundary condition in the definition of $\operatorname{dom} D$ makes sense by the Trace Theorem [34, Theorem 1.2] and takes care of (4.4).)

Before we rewrite $(4.1)$ we collect some facts about $D$ and the state spaces. For a more thorough discussion see, e.g., $[16,34]$. 


\section{Lemma 4.1.}

(a) $D$ is a densely defined, closed linear operator $X \supset \operatorname{dom} D \rightarrow Y$.

(b) Its adjoint $D^{*}$ is defined on

$$
\operatorname{dom} D^{*}=\left\{v \in H^{1}\left(\Omega, \mathbf{R}^{3}\right): v(x)=0 \text { for } x \in \Gamma_{0}\right\}
$$

by $\left(D^{*} v\right)_{i j}=\frac{1}{2}\left(v_{i, j}+v_{j, i}\right)$.

Proof. (a) As the space of test functions $\mathscr{D}\left(\Omega, \mathbf{R}_{\text {sym }}^{3 \times 3}\right)$ is contained in $\operatorname{dom} D$, it is clear that $\operatorname{dom} D$ is dense in $X$. To check closedness, assume that $\sigma^{n} \in$ $\operatorname{dom} D, \sigma^{n} \rightarrow \sigma$ in $X$, and $D \sigma^{n} \rightarrow v$ in $Y$. As $\sigma_{i j, j}^{n}$ converges to $\sigma_{i j, j}$ in the sense of distributions and to $-v_{i}$ in $L^{2}$, we infer that $\sigma_{i j, j}=-v_{i} \in L^{2}$ for $i=1, \ldots, 3$. By [34, Theorem 1.2] $0=\left.\sigma_{i j}^{n} n_{j}\right|_{\Gamma_{1}}$ converges to $\left.\sigma_{i j} n_{j}\right|_{\Gamma_{1}}$ in $H^{-1 / 2}\left(\Gamma_{1}, \mathbf{R}\right)$. Thus $\sigma \in \operatorname{dom} D$ and $D \sigma=v$.

(b) Assume first that $\sigma \in \operatorname{dom} D$ and $v \in H^{1}\left(\Omega, \mathbf{R}^{3}\right)$ with $\left.v\right|_{\Gamma_{0}}=0$. [34, (1.19)] says that for any $w \in H^{1}(\Omega, \mathbf{R})$ and $i=1, \ldots, 3$,

$$
\int_{\Omega}\left(\sigma_{i j} w_{, j}+\sigma_{i j, j} w\right) d x=\int_{\Gamma} \sigma_{i j} n_{j} w d S
$$

Utilizing symmetry of $\sigma$ we obtain

$$
\begin{aligned}
\int_{\Omega} \sigma_{i j} & \frac{1}{2}\left(v_{i, j}+v_{j, i}\right) d x+\int_{\Omega} \sigma_{i j, j} v_{i} d x \\
= & \frac{1}{2} \int_{\Omega}\left(\sigma_{i j} v_{i, j}+\sigma_{j i} v_{j, i}+\sigma_{i j, j} v_{i}+\sigma_{j i, i} v_{j}\right) d x \\
= & \frac{1}{2} \int_{\Gamma}\left(\sigma_{i j} n_{j} v_{i}+\sigma_{j i} n_{i} v_{j}\right) d S=0 .
\end{aligned}
$$

Thus $v \in \operatorname{dom} D^{*}$ and $\left(D^{*} v\right)_{i}=\frac{1}{2}\left(v_{i, j}+v_{j, i}\right)$.

Conversely, assume that $v \in \operatorname{dom} D^{*}$ and $D^{*} v=\tau$. For arbitrary $\eta \in$ $\mathscr{D}\left(\Omega, \mathbf{R}^{3 \times 3}\right)$ consider the symmetrized tensor $\sigma_{i j}=\frac{1}{2}\left(\eta_{i j}+\eta_{j i}\right)$. Taking distributional derivatives of $v$ we obtain $\int_{\Omega} \eta_{i j} \frac{1}{2}\left(v_{i, j}+v_{j, i}\right) d x=\int_{\Omega} \sigma_{i j} v_{i, j} d x=$ $-\int_{\Omega} \sigma_{i j, j} v_{i} d x=\langle D \sigma, v\rangle=\int_{\Omega} \sigma_{i j} \tau_{i j} d x=\int_{\Omega} \eta_{i j} \tau_{i j} d x$ (by symmetry of $\tau$ ); thus $\frac{1}{2}\left(v_{i, j}+v_{j, i}\right)=\tau_{i j} \in L^{2}(\Omega, \mathbf{R})$. By Korn's inequality, e.g., [16, (5.31')] this implies that $v \in H^{1}\left(\Omega, \mathbf{R}^{3}\right)$. Any arbitrary $\gamma \in H^{-1 / 2}\left(\Gamma_{0}, \mathbf{R}^{3}\right)$ can be extended by 0 to $\Gamma_{1}$. By [34, Remark 1.2] there exists some $\sigma \in \operatorname{dom} D$ such that $\sigma_{i j} n_{j}=\gamma_{i}$ on $\Gamma$. Again using [34, (1.19)], we infer that $\int_{\Gamma_{0}} \gamma_{i} v_{i} d S=$ $\int_{T} \gamma_{i} v_{i} d S=\int_{\Omega}\left(\sigma_{i j} v_{i, j}+\sigma_{i j, j} v_{i}\right) d x=\left\langle\sigma, D^{*} v\right\rangle-\langle D \sigma, v\rangle=0$. As $\gamma$ was arbitrary, we infer that $\left.v\right|_{\Gamma_{0}}=0$. Thus $v$ satisfies $v \in H^{1}\left(\Omega, \mathbf{R}^{3}\right)$ and $v(x)=0$ for $x \in \Gamma_{0}$, and $\left(D^{*} v\right)_{i j}=\tau_{i j}=\frac{1}{2}\left(v_{i, j}+v_{j, i}\right)$.

In the context of our viscoelastic problem this says that $\varepsilon^{\prime}=D^{*} v$. Defining the operator $A(t): X \rightarrow X$ by $(A(t) \varepsilon)_{i j}(x)=a_{i j k l}(t) \varepsilon_{k l}(x)$, we can now rewrite (4.2) as

$$
\sigma(t)=\int_{-\infty}^{t} A(t-s) D^{*} v(s) d s
$$


Hypothesis A can easily be derived from Hypothesis a. Thus (2.1), (2.2) serve as an abstract setting for (4.1)-(4.4). The following theorems are literal translations of Corollaries 2.2 and 3.2.

Theorem 4.2.

(a) If $v:(-\infty, 0] \rightarrow H^{1}\left(\Omega, \mathbf{R}^{3}\right)$ is such that $\left.v\right|_{\Gamma_{0}}=0$ and $\phi_{0}(\zeta,)=$, $\int_{-\infty}^{0} e^{\zeta t} G^{1 / 2}(\zeta) D^{*} v(t) d t$ satisfies

$$
\begin{gathered}
\phi_{0} \in L_{\nu}^{2}\left((0, \infty), L^{2}\left(\Omega, \mathbf{R}_{\mathrm{sym}}^{3 \times 3}\right)\right), \\
\zeta \phi_{0}-G^{1 / 2}(\zeta) D^{*} v(0) \in L_{\nu}^{2}\left((0, \infty), L^{2}\left(\Omega, \mathbf{R}_{\mathrm{sym}}^{3 \times 3}\right)\right),
\end{gathered}
$$

and $\int_{-\infty}^{0} a(-t) D^{*} v(t) d t \in \operatorname{dom} D$, then there exist $v \in C^{1}\left([0, \infty), L^{2}\left(\Omega, \mathbf{R}^{3}\right)\right)$, $\sigma \in C\left((0, \infty), L^{2}\left(\Omega, \mathbf{R}_{\mathrm{sym}}^{3 \times 3}\right)\right)$, such that (4.1)-(4.4) are satisfied.

(b) If $v^{n}:(-\infty, 0) \rightarrow \operatorname{dom} D^{*}$ satisfy the conditions above and converge in the sense that $v^{n}(0) \rightarrow v_{0} \in Y$ and $\phi_{0}^{n} \rightarrow \phi_{0} \in L_{\nu}^{2}$, then the solutions $v^{n}$ converge to some $v \in C\left([0, \infty), L^{2}\left(\Omega, \mathbf{R}^{3}\right)\right)$ uniformly on compact intervals. We call $v$ the generalized solution to (4.1)-(4.4).

Theorem 4.3. Let $i \beta \hat{a}(i \beta)=u(i \beta)+i v(i \beta)$ with complex fourth-order tensors satisfying $u_{i j k l}=u_{j i k l}=\bar{u}_{k l i j}$ and $v_{i j k l}=v_{j i k l}=v_{k l i j}$. Let $\gamma:(0, \infty) \rightarrow(0, \infty)$ be such that $\beta v(i \beta)-\gamma(\beta) u(i \beta)$ is positive semidefinite in the sense that for each second-order tensor $\left(\xi_{i j}\right), \bar{\xi}_{i j}\left[\beta v_{i j k l}(i \beta)-\gamma(\beta) u_{i j k l}(i \beta)\right] \xi_{k l} \geq 0$.

(a) If $\lim \sup _{\beta \rightarrow \infty} \log (\beta) / \gamma(\beta)=0$, then the generalized solution $v$ to (4.1)(4.4) is in $C^{\infty}\left((0, \infty), H^{1}\left(\Omega, \mathbf{R}^{3}\right)\right)$, and an associated $\sigma \in C^{\infty}((0, \infty), \operatorname{dom} D)$ is defined.

(b) If lim sup $\sin _{\beta \rightarrow \infty} \log (\beta) / \gamma(\beta)<\infty$, then $v$ gains smoothness in time gradually. For sufficiently large $t>0 \quad v(t) \in \operatorname{dom} D^{*}$, and $\sigma(t)$ is defined, gaining smoothness gradually.

(c) If $\lim \sup _{\beta \rightarrow \infty} \beta / \gamma(\beta)<\infty$, then $v$ is analytic in $t$. (In particular, disturbances are propagated in space with infinite speed.)

We apply these results to a linearly viscoelastic, isotropic medium. By isotropy the tensor $a$ has the form, e.g., $[15,(8.4-4)]$

$$
a_{i j k l}(t)=\lambda(t) \delta_{i j} \delta_{k l}+\mu(t)\left[\delta_{i k} \delta_{j l}+\delta_{i l} \delta_{j k}\right]
$$

$\lambda$ and $\mu$ are the Lamé moduli, $\mu$ is the shear modulus, and $\kappa=\lambda+2 \mu / 3$ is the bulk modulus. We assume that both $\kappa$ and $\mu$ are completely monotonic.

Keeping in mind that we consider only action of $a$ on symmetric $3 \times 3$ tensors, we rewrite $a$ as a $6 \times 6$ matrix with respect to the basis

$$
\begin{array}{lll}
e_{1}=\left(\begin{array}{lll}
1 & 0 & 0 \\
0 & 0 & 0 \\
0 & 0 & 0
\end{array}\right), & e_{2}=\left(\begin{array}{lll}
0 & 0 & 0 \\
0 & 1 & 0 \\
0 & 0 & 0
\end{array}\right), & e_{3}=\left(\begin{array}{lll}
0 & 0 & 0 \\
0 & 0 & 0 \\
0 & 0 & 1
\end{array}\right), \\
e_{4}=\left(\begin{array}{lll}
0 & \frac{1}{2} & 0 \\
\frac{1}{2} & 0 & 0 \\
0 & 0 & 0
\end{array}\right), & e_{5}=\left(\begin{array}{lll}
0 & 0 & \frac{1}{2} \\
0 & 0 & 0 \\
\frac{1}{2} & 0 & 0
\end{array}\right), & e_{6}=\left(\begin{array}{lll}
0 & 0 & 0 \\
0 & 0 & \frac{1}{2} \\
0 & \frac{1}{2} & 0
\end{array}\right),
\end{array}
$$


of $\mathbf{R}_{\mathrm{sym}}^{3 \times 3}$ and obtain

$$
a \cong\left(\begin{array}{cccccc}
\lambda+2 \mu & \lambda & \lambda & 0 & 0 & 0 \\
\lambda & \lambda+2 \mu & \lambda & 0 & 0 & 0 \\
\lambda & \lambda & \lambda+2 \mu & 0 & 0 & 0 \\
0 & 0 & 0 & 2 \mu & 0 & 0 \\
0 & 0 & 0 & 0 & 2 \mu & 0 \\
0 & 0 & 0 & 0 & 0 & 2 \mu
\end{array}\right) .
$$

A simple orthogonal similarity transform transforms this matrix into

$$
\operatorname{diag}(3 \lambda+2 \mu, 2 \mu, 2 \mu, 2 \mu, 2 \mu, 2 \mu)=\operatorname{diag}(3 \kappa, 2 \mu, 2 \mu, 2 \mu, 2 \mu, 2 \mu) .
$$

Hence

$$
\begin{aligned}
& u(i \beta) \cong \operatorname{diag}(\operatorname{Re}(3 i \beta \hat{\kappa}(i \beta)), \operatorname{Re}(2 i \beta \hat{\mu}(i \beta), \ldots), \\
& v(i \beta) \cong \operatorname{diag}(\operatorname{Im}(3 i \beta \hat{\kappa}(i \beta)), \operatorname{Im}(2 i \beta \hat{\mu}(i \beta), \ldots)
\end{aligned}
$$

with respect to a suitable basis in $\mathbf{R}_{\text {sym }}^{3 \times 3}$. Consequently $\gamma$ may be defined by

$$
\gamma(\beta)=\min \left(-\frac{\beta \operatorname{Re} \hat{\kappa}(i \beta)}{\operatorname{Im} \hat{\kappa}(i \beta)},-\frac{\beta \operatorname{Re} \hat{\mu}(i \beta)}{\operatorname{Im} \hat{\mu}(i \beta)}\right) .
$$

We therefore obtain

Corollary 4.4. Consider a linearly viscoelastic isotropic body where the bulk modulus $\kappa$ and the shear modulus $\mu$ are completely monotonic.

(a) If both

$$
\limsup _{\beta \rightarrow \infty}-\frac{\log \beta \operatorname{Im} \hat{\kappa}(i \beta)}{\beta \operatorname{Re} \hat{\kappa}(i \beta)}=0
$$

and

$$
\limsup _{\beta \rightarrow \infty}-\frac{\log \beta \operatorname{Im} \hat{\mu}(i \beta)}{\beta \operatorname{Re} \hat{\mu}(i \beta)}=0,
$$

then $v(t)$ is infinitely differentiable in time, and $\sigma(t)$ is defined and infinitely differentiable in time for all $t>0$.

(b) If both

$$
\limsup _{\beta \rightarrow \infty}-\frac{\log \beta \operatorname{Im} \hat{\kappa}(i \beta)}{\beta \operatorname{Re} \hat{\kappa}(i \beta)}<\infty .
$$

and

$$
\limsup _{\beta \rightarrow \infty}-\frac{\log \beta \operatorname{Im} \hat{\mu}(i \beta)}{\beta \operatorname{Re} \hat{\mu}(i \beta)}<\infty,
$$

then $v(t)$ is differentiable in time, and $\sigma(t)$ is defined for sufficiently large $t>0$. With increasing $t, v$ and $\sigma$ gain smoothness.

(c) If both

$$
\limsup _{\beta \rightarrow \infty}-\frac{\operatorname{Im} \hat{\kappa}(i \beta)}{\operatorname{Re} \hat{\kappa}(i \beta)}<\infty
$$

and

$$
\limsup _{\beta \rightarrow \infty}-\frac{\operatorname{Im} \hat{\mu}(i \beta)}{\operatorname{Re} \hat{\mu}(i \beta)}<\infty
$$

then $v(t)$ is analytic in $t$. 


\section{THERMOVISCOELASTICITY}

We modify the methods above to treat certain cases of linear thermoviscoelasticity. In [5, p. 89f] we find the following general equations:

$$
\begin{gathered}
\rho v_{i}^{\prime}(t, x)=\sigma_{i j, j}(t, x), \\
\sigma_{i j}(t, x)=\int_{-\infty}^{t} a_{i j k l}(t-\tau) \varepsilon_{k l}^{\prime}(\tau, x) d \tau-\int_{-\infty}^{t} b_{i j}(t-\tau) \theta^{\prime}(\tau, x) d \tau,
\end{gathered}
$$

$$
k_{i j} \theta_{, i j}(t, x)=\frac{\partial}{\partial t}\left[\int_{-\infty}^{t} m(t-\tau) \theta^{\prime}(\tau, x) d \tau+\int_{-\infty}^{t} b_{i j}(t-\tau) \varepsilon_{i j}^{\prime}(\tau, x) d \tau\right],
$$

where $\theta$ denotes the temperature field, $k$ and $m$ are physical quantities governing heat conduction, and $b$ describes conversion of mechanical energy to heat and vice versa. The other parameters have the same meaning as in the previous section. (As we do not necessarily assume that the material is in stress-free configuration at time 0 , we integrate from $-\infty$ to $t$ in the convolution terms. We assume no body forces. Some of the coefficients have been renamed and rescaled.)

In addition to the mechanical boundary conditions of the previous section,

$$
\begin{gathered}
v_{i}(t, x)=0 \text { on } \Gamma_{0}, \\
\sigma_{i j}(t, x) n_{j}(x)=0 \quad \text { on } \Gamma_{1},
\end{gathered}
$$

we assume that there is complete heat insulation on one part of the boundary and constant temperature 0 on its complement.

$$
\begin{gathered}
\theta(t, x)=0 \text { on } \Gamma_{3}, \\
k_{i j} n_{j}(x) \theta_{, j}(t, x)=0 \quad \text { on } \Gamma_{4},
\end{gathered}
$$

where $\Gamma=\Gamma_{3}+\Gamma_{4}$ is another partition of the boundary.

We simplify the equations above by assuming that there are no memory effects in the law of heat conduction. This yields

$$
\begin{gathered}
\sigma_{i j}(t, x)=\int_{-\infty}^{t} a_{i j k l}(t-\tau) \varepsilon_{k l}^{\prime}(\tau, x) d \tau-b_{i j} \theta(t, x), \\
m \theta^{\prime}(t, x)=k_{i j} \theta_{, i j}(t, x)-b_{i j} \varepsilon_{i j}^{\prime}(t, x) .
\end{gathered}
$$

Besides the hypotheses of $\S 4$ we require

Hypothesis $\mathrm{k} .\left(k_{i j}\right)$ is a symmetric positive definite real $3 \times 3$ tensor, $\left(b_{i j}\right)$ is a real $3 \times 3$ tensor, and $m>0$. 
The state spaces for $v(t,$.$) and \sigma(t,$.$) are again Y=L^{2}\left(\Omega, \mathbf{R}^{3}\right)$ and $X=$ $L^{2}\left(\Omega, \mathbf{R}_{\text {sym }}^{3 \times 3}\right)$, respectively. For $\theta(t,$.$) we consider the space Z=L^{2}(\Omega, \mathbf{R})$. The equations can now be rewritten

$$
\begin{gathered}
R v^{\prime}(t)=-D \sigma(t), \\
\sigma(t)=\int_{-\infty}^{t} A(t-s) D^{*} v(s) d s+B \theta(t), \\
M \theta^{\prime}(t)=-L \theta(t)+B^{*} D^{*} v(t) .
\end{gathered}
$$

$R, A, D, D^{*}$ are as before. $M$ is just multiplication by $m$ but can be any positive definite continuously invertible operator in $Z$. $L: Z \supset \operatorname{dom} L \rightarrow Z$ is a positive semidefinite self-adjoint operator, namely $L \theta=-k_{i j} \theta_{, i j}$, defined on

$$
\operatorname{dom} L=\left\{\theta \in H^{2}(\Omega, \mathbf{R}): \theta(x)=0 \text { on } \Gamma_{3}, k_{i j} \theta_{, i} n_{j}=0 \text { on } \Gamma_{4}\right\},
$$

and $B$ is a bounded linear operator $Z \rightarrow X$, namely $(B \theta)_{i j}=-b_{i j} \theta$.

We again construct a semigroup as in $\S 2$. Including $\theta$ we obtain a state $(v, \phi, \theta) \in \mathscr{Z}=Y \times L_{\nu}^{2}((0, \infty), X) \times Z$, normed by

$$
\|(v, \phi, \theta)\|^{2}=\langle v, R v\rangle+\int_{0}^{\infty}\langle\phi(\zeta), \phi(\zeta)\rangle d \nu(\zeta)+\langle\theta, M \theta\rangle .
$$

The abstract differential equation is now

$$
\begin{gathered}
v^{\prime}(t)=-R^{-1} D\left[\int_{0}^{\infty} G^{1 / 2}(\zeta) \phi(t, \zeta) d \nu(\zeta)+B \theta(t)\right], \\
\phi^{\prime}(t, \zeta)=G^{1 / 2}(\zeta) D^{*} v(t)-\zeta \phi(t, \zeta), \\
\theta^{\prime}(t)=-M^{-1}\left[L \theta(t)-B^{*} D^{*} v(t)\right]
\end{gathered}
$$

So we define an operator $\mathscr{B}$ on

$$
\begin{array}{r}
\operatorname{dom} \mathscr{B}=\left\{(v, \phi, \theta) \in \mathscr{Z}: v \in \operatorname{dom} D^{*}, G^{1 / 2}(\zeta) D^{*} v-\zeta \phi \in L_{\nu}^{2}, \theta \in \operatorname{dom} L,\right. \\
\\
\left.\int_{0}^{\infty} G^{1 / 2}(\zeta) \phi(\zeta) d \nu(\zeta)+B \theta \in \operatorname{dom} D\right\}
\end{array}
$$

by

$$
\begin{aligned}
\mathscr{B}(v, \phi, \theta)=\left(-R^{-1} D\left[\int_{0}^{\infty} G^{1 / 2}(\zeta) \phi(\zeta) d \nu(\zeta)+B \theta\right],\right. \\
\left.D^{*} v-\zeta \phi(\zeta),-M^{-1}\left[L \theta-B^{*} D^{*} v\right]\right) .
\end{aligned}
$$

We again obtain the wellposedness result:

Theorem 5.1. $\mathscr{B}$ generates a semigroup $\mathscr{T}(t)$ of contractions in $\mathscr{Z}$.

Proof. The proof is very similar to the proof of Theorem 2.1, so we state only the necessary modifications. To prove that $\mathscr{B}$ is densely defined, proceed as in the proof of Lemma 2.5. To approximate $(y, \psi, \eta) \in \mathscr{Z}$ by $(v, \phi, \theta) \in$ $\operatorname{dom} \mathscr{B}$, first choose $v \in \operatorname{dom} D^{*}$ close to $y$ and $\theta \in \operatorname{dom} L$ close to $\eta$. Then 
construct $\pi$ and $\psi$ as in Lemma 2.5 , but such that $\int_{0}^{\infty} G^{1 / 2}(\zeta) \phi(\zeta) d \nu(\zeta)+$ $B \theta \in \operatorname{dom} D$.

Direct computation as in Lemma 2.6 shows that for $(v, \phi, \theta) \in \operatorname{dom} \mathscr{B}$

$$
\operatorname{Re}\langle(v, \phi, \theta), \mathscr{B}(v, \phi, \theta)\rangle=-\operatorname{Re}\left[\int_{0}^{\infty} \zeta\langle\phi(\zeta), \phi(\zeta)\rangle d \nu(\zeta)+\langle\theta, L \theta\rangle\right] \leq 0 ;
$$

thus $\mathscr{B}$ is dissipative.

Now fix some $\lambda>0$. As in Lemma 2.7, we show that $(\lambda-\mathscr{B})^{-1}$ exists. Again, all we have to do is to find some solution $(v, \phi, \theta)$ to

$$
\begin{gathered}
\lambda v+R^{-1} D\left[\int_{0}^{\infty} G^{1 / 2}(\zeta) \phi(\zeta) d \nu(\zeta)+B \theta\right]=y, \\
(\lambda+\zeta) \phi(\zeta)-G^{1 / 2}(\zeta) D^{*} v=\psi(\zeta) \\
\lambda \theta+M^{-1}\left(L \theta-B^{*} D^{*} v\right)=\eta
\end{gathered}
$$

We solve explicitly for $\phi$ and $\theta$ in terms of $v$ :

$$
\phi(\zeta)=\frac{1}{\lambda+\zeta}\left(\psi(\zeta)+G^{1 / 2}(\zeta) D^{*} v\right), \theta=\left(\lambda+M^{-1} L\right)^{-1}\left(\eta+M^{-1} B^{*} D^{*} v\right) .
$$

$\left(\lambda+M^{-1} L\right)^{-1}$ exists, since $M^{-1} L$ is self-adjoint positive semidefinite with respect to the scalar product $\langle z, M z\rangle$. With $\tilde{\psi}$ as in $\S 2$ and

$$
z=\int_{0}^{\infty} G^{1 / 2}(\zeta) \phi(\zeta) d \nu(\zeta)+B \theta
$$

we have to solve

$$
\begin{gathered}
\lambda v+R^{-1} D z=y, \\
\lambda z-\lambda\left[\widehat{A}(\lambda)+B\left(\lambda+M^{-1} L\right)^{-1} M^{-1} B^{*}\right] D^{*} v=\lambda \tilde{\psi}(\lambda)+\lambda B\left(\lambda+M^{-1} L\right)^{-1} \eta .
\end{gathered}
$$

This is just the system (2.7), (2.8) with a modified right-hand side and $\widehat{A}(\lambda)$ replaced by the operator $\widehat{A}(\lambda)+B(\lambda M+L)^{-1} B^{*}$, which is positive definite and continuously invertible as well. Thus the proof of Lemma 2.7 shows that there exists a solution.

In the thermoviscoelastic case the following version of the smoothing criterion holds:

Theorem 5.2. Let $\mathscr{T}(t)$ be the semigroup generated by $\mathscr{B}$, and

$$
\lambda\left[\widehat{A}(\lambda)+B(\lambda M+L)^{-1} B^{*}\right]=\widetilde{U}(\lambda)+i \widetilde{V}(\lambda)
$$

with self-adjoint operators $\widetilde{U}$ and $\widetilde{V}$. Suppose that $\gamma:(0, \infty) \rightarrow(0, \infty)$ is such that for all $\beta>0, \beta \widetilde{V}(i \beta)-\gamma(\beta) \widetilde{U}(i \beta)$ is positive semidefinite.

(a) If $\lim \sup _{\beta \rightarrow \infty} \log (\beta) / \gamma(\beta)=0$, then $\mathscr{T}(t)$ is differentiable.

(b) If $\lim \sup _{\beta \rightarrow \infty} \log (\beta) / \gamma(\beta)<\infty$, then $\mathscr{T}(t)$ is eventually differentiable.

(c) If $\lim \sup _{\beta \rightarrow \infty} \beta / \gamma(\beta)<\infty$, then $\mathscr{T}(t)$ is analytic.

For the proof we need a simple technical lemma: 
Lemma 5.3. If $H$ is a (possibly unbounded) self-adjoint operator in a Hilbert space $\mathscr{H}$, and $x \in \mathscr{H}$, then for any real $\beta,\left\langle(-i \beta+H)^{-1} x,(-i \beta+H)^{-1} x\right\rangle=$ $(1 / \beta) \operatorname{Im}\left\langle x,(i \beta+H)^{-1} x\right\rangle$.

Proof.

$$
\begin{aligned}
& \left\langle(-i \beta+H)^{-1} x,(-i \beta+H)^{-1} x\right\rangle=\left\langle x,(i \beta+H)^{-1}(-i \beta+H)^{-1} x\right\rangle \\
& \quad=(1 / 2 i \beta)\left\langle x,(-i \beta+H)^{-1} x-(i \beta+H)^{-1} x\right\rangle \\
& =(1 / \beta) \operatorname{Im}\left\langle x,(i \beta+H)^{-1} x\right\rangle .
\end{aligned}
$$

Proof of Theorem 5.2. We proceed as in the proof of Theorem 3.1. There the crucial part was Lemma 3.4. So we show again (and in the same fashion) that $\left\|(i \beta-\mathscr{B})^{-1}\right\|=O(1 / \gamma(\beta)+1 / \beta)$ as $\beta \rightarrow \infty$.

Assume that $(i \beta-\mathscr{B})(v, \phi, \theta)=(y, \psi, \eta)$ with $\|(y, \psi, \eta)\| \leq 1$, i.e.,

$$
\begin{gathered}
i \beta v+R^{-1} D\left[\int_{0}^{\infty} G^{1 / 2}(\zeta) \phi(\zeta) d \nu(\zeta)+B \theta\right]=y, \\
i \beta \theta(\zeta)-G^{1 / 2}(\zeta) D^{*} v(\zeta)+\zeta \phi(\zeta)=\psi(\zeta), \\
i \beta \theta+M^{-1}\left(L \theta-B^{*} D^{*} v\right)=\eta .
\end{gathered}
$$

We solve explicitly

$$
\phi(\zeta)=\frac{1}{\zeta+i \beta} \psi(\zeta)+\frac{1}{\zeta+i \beta} G^{1 / 2}(\zeta) D^{*} v,
$$

hence

$$
\int_{0}^{\infty} G^{1 / 2}(\zeta) \phi(\zeta) d \nu(\zeta)=\tilde{\psi}(i \beta)+\widehat{A}(i \beta) D^{*} v
$$

with $\tilde{\psi}$ as in Lemma 3.4 , and

$$
\theta=\left(i \beta+M^{-1} L\right)^{-1} \eta+\left(i \beta+M^{-1} L\right)^{-1} M^{-1} B^{*} D^{*} v .
$$

Notice that $M^{-1} L$ is self-adjoint with respect to the inner product $\langle x, M y\rangle$, so that $\left(i \beta+M^{-1} L\right)^{-1}$ exists. Now (5.9) becomes

$$
\begin{aligned}
i \beta v+R^{-1} D\left\{\left[\hat{A}(i \beta)+B\left(i \beta+M^{-1} L\right)^{-1} M^{-1} B^{*}\right] D^{*} v\right. \\
\left.+\left[\tilde{\psi}(i \beta)+B\left(i \beta+M^{-1} L\right)^{-1} \eta\right]\right\}=y .
\end{aligned}
$$

This equation should be compared to (3.4). The operator $\widehat{A}(i \beta)$ has been replaced by $\hat{A}(i \beta)+B\left(i \beta+M^{-1} L\right)^{-1} M^{-1} B^{*}$, and $\tilde{\psi}(i \beta)$ has changed into $\tilde{\psi}(i \beta)+B\left(i \beta+M^{-1} L\right)^{-1} \eta$. As $\tilde{U}$ and $\tilde{V}$ play the same role for $\widehat{A}(i \beta)+$ $B\left(i \beta+M^{-1} L\right)^{-1} M^{-1} B^{*}$ as $U$ and $V$ play for $\widehat{A}(i \beta)$, we can get the estimates

$$
\begin{gathered}
\left\langle D^{*} v, \tilde{U} D^{*} v\right\rangle^{1 / 2}=O(\beta / \gamma(\beta)+1), \\
\|v\|=O(1 / \gamma(\beta)+1 / \beta),
\end{gathered}
$$

much as (3.12) and (3.13) once we can show that for any $x \in X$

$$
\left|\left\langle x, \tilde{\psi}(i \beta)+B\left(i \beta+M^{-1} L\right)^{-1} \eta\right\rangle\right|=O\left(\beta^{-1}\right)\langle x, \widetilde{U} x\rangle^{1 / 2}
$$


(cf. (3.9)). Since

$$
\begin{aligned}
\langle x, \widetilde{U} x-U x\rangle & =\frac{1}{2}\left\langle x, B\left[i \beta\left(i \beta+M^{-1} L\right)^{-1}-i \beta\left(-i \beta+M^{-1} L\right)^{-1}\right] M^{-1} B^{*} x\right\rangle \\
& =\frac{1}{2}\left\langle x, \beta^{2} B\left(i \beta+M^{-1} L\right)^{-1}\left(-i \beta+M^{-1} L\right)^{-1} M^{-1} B^{*} x\right\rangle \\
& =\frac{\beta^{2}}{2}\left\langle\left(-i \beta+M^{-1} L\right)^{-1} M^{-1} B^{*} x, M\left(-i \beta+M^{-1} L\right)^{-1} M^{-1} B^{*} x\right\rangle \\
& \geq 0,
\end{aligned}
$$

we obtain from (3.9) that $|\langle x, \tilde{\psi}(i \beta)\rangle|=O\left(\beta^{-1}\right)\langle x, \widetilde{U} x\rangle^{1 / 2}$.

$$
\begin{aligned}
& \left|\left\langle x, B\left(i \beta+M^{-1} L\right)^{-1} \eta\right\rangle\right|=\left|\left\langle\left(-i \beta+M^{-1} L\right)^{-1} M^{-1} B^{*} x, M \eta\right\rangle\right| \\
& \quad \leq\langle\eta, M \eta\rangle^{1 / 2}\left\langle\left(-i \beta+M^{-1} L\right)^{-1} M^{-1} B^{*} x, M\left(-i \beta+M^{-1} L\right)^{-1} M^{-1} B^{*} x\right\rangle^{1 / 2} .
\end{aligned}
$$

Lemma 5.3, applied to $H=M^{-1} L$ and inner product $\langle x, M y\rangle$ yields

$$
\begin{aligned}
\mid\langle x, & \left.B\left(i \beta+M^{-1} L\right)^{-1} \eta\right\rangle \mid \\
& \leq O\left(\beta^{-1 / 2}\right)\left(\operatorname{Im}\left\langle M^{-1} B^{*} x, M\left(i \beta+M^{-1} L\right)^{-1} M^{-1} B^{*} x\right\rangle\right)^{1 / 2} \\
& =O\left(\beta^{-1 / 2}\right)\left(\operatorname{Re}\left\langle x, i B\left(\left(i \beta+M^{-1} L\right)^{-1} M^{-1} B^{*} x\right\rangle\right)^{1 / 2}=O\left(\beta^{-1}\right)\langle x, \widetilde{U} x\rangle^{1 / 2} .\right.
\end{aligned}
$$

Consequently (5.15) holds and the estimates (5.13) and (5.14) for $v$ are valid. Now $\phi$ can be estimated precisely as in Lemma (3.4). To estimate $\|\theta\|$ notice that $\left\|\left(i \beta+M^{-1} L\right)^{-1} \eta\right\|=O\left(\beta^{-1}\right)$, since $M^{-1} L$ is self-adjoint with respect to a suitable scalar product, and that (5.16) backward reads

$$
\begin{aligned}
\left\langle\left(i \beta+M^{-1} L\right)^{-1}\right. & \left.M^{-1} B^{*} D^{*} v, M\left(i \beta+M^{-1} L\right)^{-1} M^{-1} B^{*} D^{*} v\right\rangle^{1 / 2} \\
& \leq \frac{2}{\beta}\left\langle D^{*} v,(\widetilde{U}-U) D^{*} v\right\rangle^{1 / 2}=O(1 / \gamma(\beta)+1 / \beta) \text { by (5.13). }
\end{aligned}
$$

Acknowledgements. This work was begun while W. D. was visiting Southern Illinois University at Carbondale and finished while R. G. was visiting Universität Graz, both visits within the Austrian-American Cooperative Research Program, FWF grant P-5691 and NSF grant DMS-8701552, respectively. It is a pleasure to thank Southern Illinois University and Universität Graz for kind hospitality, and the National Science Foundation and Fonds zur Förderung der Wissenschaftlichen Forschung for their support. Moreover, W. D. is indebted to R. Wheeler, K. Hannsgen, and J. U. Kim for many helpful and stimulating discussions during a visit of W. D. to Virginia Tech. supported by the Air Force Office of Scientific Research, AFSOR-86-0085. Also, we thank J. Prüss for providing his recent manuscript [30] and M. Renardy for insight in the new monograph [32].

\section{REFERENCES}

1. J. Achenbach and D. Reddy, Note on wave propagation in linearly viscoelastic media, Z. Angew. Math. Phys. 18 (1967), 141-144.

2. R. L. Bagley and P. J. Torvik, Fractional calculus, a different approach to viscoelastically damped structures, AIAA J. 21 (1983), 741-748. 
3. M. Caputo and F. Mainardi, Linear models of dissipation in anelastic solids, Riv. Nuovo Cimento (2) 1 (1971), 161-198.

4. G. Chen and R. C. Grimmer, Semigroups and integral equations, J. Integral Equations 2 (1980), 133-154.

5. R. M. Christensen, Theory of viscoelasticity. An introduction, 2nd ed., Academic Press, 1982.

6. B. T. Chu, Stress waves in isotropic linear viscoelastic materials, J. Mécanique 1 (1962), 439462.

7. B. D. Coleman and M. E. Gurtin, Waves in materials with memory II. On the growth and decay of one-dimensional acceleration waves, Arch. Rational Mech. Anal. 19 (1965), 239-265.

8. C. M. Dafermos, Contraction semigroups and trend to equilibrium in continuum mechanics, IUTAM/IMU Sympos. on Applications of Methods of Functional Analysis to Problems in Mechanics (P. Germain and P. Nayroles, eds.), Lecture Notes in Math., vol. 503, Springer, Berlin, 1976.

9. W. Desch and R. C. Grimmer, Propagation of singularities for integrodifferential equations, J. Differential Equations 65 (1986), 411-426.

10. __ Initial-boundary value problems for integrodifferential equations, J. Integral Equations 10 (1985), 73-97.

11. __ Smoothing properties of linear Volterra integrodifferential equations, SIAM J. Math. Anal. 20 (1989), 116-132.

12. W. Desch, R. C. Grimmer, and W. Schappacher, Propagation of singularities by solutions of second order integrodifferential equations (to appear).

13. W. Desch and R. K. Miller, Exponential stabilization of Volterra integral equations with singular kernels (in preparation).

14. J. D. Ferry, Viscoelastic properties of polymers, 2nd ed., Wiley, New York, 1970.

15. Y. C. Fung, A first course in continuum mechanics, 2nd ed., Prentice-Hall, Englewood Cliffs, N. J., 1977.

16. V. Girault and P. A. Raviart, Finite element methods for Navier-Stokes equations. Theory and algorithms, Springer, Berlin, 1986.

17. D. Graff, Mathematical models and waves in linear viscoelasticity, Wave Propagation in Viscoelastic Media (F. Mainardi, ed.), Res. Notes in Math., 52, Pitman, London, 1982, pp. 1-27.

18. R. C. Grimmer and A. J. Pritchard, Analytic resolvent operators for integral equations in Banach space, J. Differential Equations 50 (1983), 234-259.

19. K. Hannsgen and R. L. Wheeler, Behavior of the solution of a Volterra equation as a parameter tends to infinity, J. Integral Equations 7 (1984), 229-237.

20. K. Hannsgen, Y. Renardy, and R. L. Wheeler, Effectiveness and robustness with respect to time delays of boundary feedback stabilization in one-dimensional viscoelasticity, SIAM J. Control Optim. 26 (1988), 1200-1234.

21. W. J. Hrusa and M. Renardy, On wave propagation in linear viscoelasticity, Quart. Appl. Math. 43 (1985), 237-254.

22. J. A. Hudson, The excitation and propagation of elastic waves, Cambridge Univ. Press, London, 1980.

23. J. Kazakia and R. S. Rivlin, Run-up and spin-up in a viscoelastic fluid I, Rheol. Acta 20 (1981), 111-127.

24. R. K. Miller, Volterra integral equations in a Banach space, Funkcial. Ekvac. 18 (1975), 163194.

25. A. Narain and D. D. Joseph, Linearlized dynamics for step jumps in velocity and displacement of shearing flows of a simple fluid, Rheol. Acta 21 (1982), 228-250.

26. _ Classification of linear viscoelastic solids based on a failure criterion, J. Elasticity 14 (1984), 19-26. 
27. A. Pazy, On the differentiability and compactness of semigroups of linear operators, J. Math. Mech. 17 (1968), 1131-1141.

28. __ Semigroups of linear operators and applications to linear partial differential equations, Springer, Berlin, 1983.

29. J. Prüss, Positivity and regularity of hyperbolic Volterra equations in Banach spaces, Math. Ann. 279 (1987), 317-344.

30. __ Regularity and integrability of resolvents of linear Volterra equations, Proc. Conf. on Volterra Integral Equations in Banach Spaces and Applications, Trento, 1987 (to appear).

31. M. Renardy, Some remarks on the propagation and nonpropagation of discontinuities in linearly viscoelastic liquids, Rheol. Acta 21 (1982), 251-254.

32. M. Renardy, W. J. Hrusa, and J. A. Nohel, Mathematical problems in viscoelasticity, Longman, 1987.

33. M. Slemrod, $A$ hereditary partial differential equation with applications in the theory of simple fluids, Arch. Rational Mech. Anal. 62 (1976), 303-322.

34. R. Temam, Navier-Stokes equations. Theory and numerical analysis, rev. ed., North-Holland, Amsterdam, 1979.

35. J. N. Welch, On the construction of the Hilbert space $L_{2, M}$ for an operator valued measure $M$, Vector and Operator Valued Measures and Applications (D. H. Tucker and H. B. Maynard, eds.), Academic Press, New York, 1973.

Institut für Mathematik, Universität Graz, A-8010 Graz, Austria

Department of Mathematics, Southern Illinois University, Carbondale, Illinois 62901 